\title{
Sequential Hydrogenation of $\mathrm{CO}_{2}$ to Methanol Using a Pincer Iron Catalyst
}

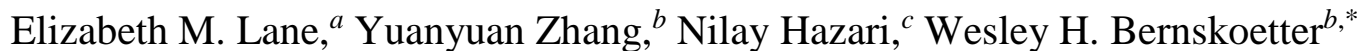

${ }^{a}$ The Department of Chemistry, Brown University, Providence, Rhode Island, 02912, USA; ${ }^{\text {bThe }}$ Department of Chemistry, The University of Missouri, Columbia, Missouri, 65211, USA; ${ }^{\circ}$ The

Department of Chemistry, Yale University, P. O. Box 208107, New Haven, Connecticut, 06520, USA.

Procedure for Synthesizing Morphylammonium Morphylcarbamate $\mathrm{S} 2$ Optimization Tables for $\mathrm{CO}_{2}$ Hydrogenation 


\section{Procedure for Synthesizing Morphylammonium Morphylcarbamate:}

Morpholine and tetrahydrofuran (THF) were transferred into a glass Parr insert and sealed in a reactor. The vessel was pressurized with $1000 \mathrm{psi} \mathrm{CO}_{2}$ and stirred for 16 hours at $120{ }^{\circ} \mathrm{C}$. After cooling reactor in an ice bath for 45 minutes, it was vented to the air and the resulting white solid collected by filtration. X-ray quality crystals were obtained from a highly concentrated solution in 1,4-dioxane.
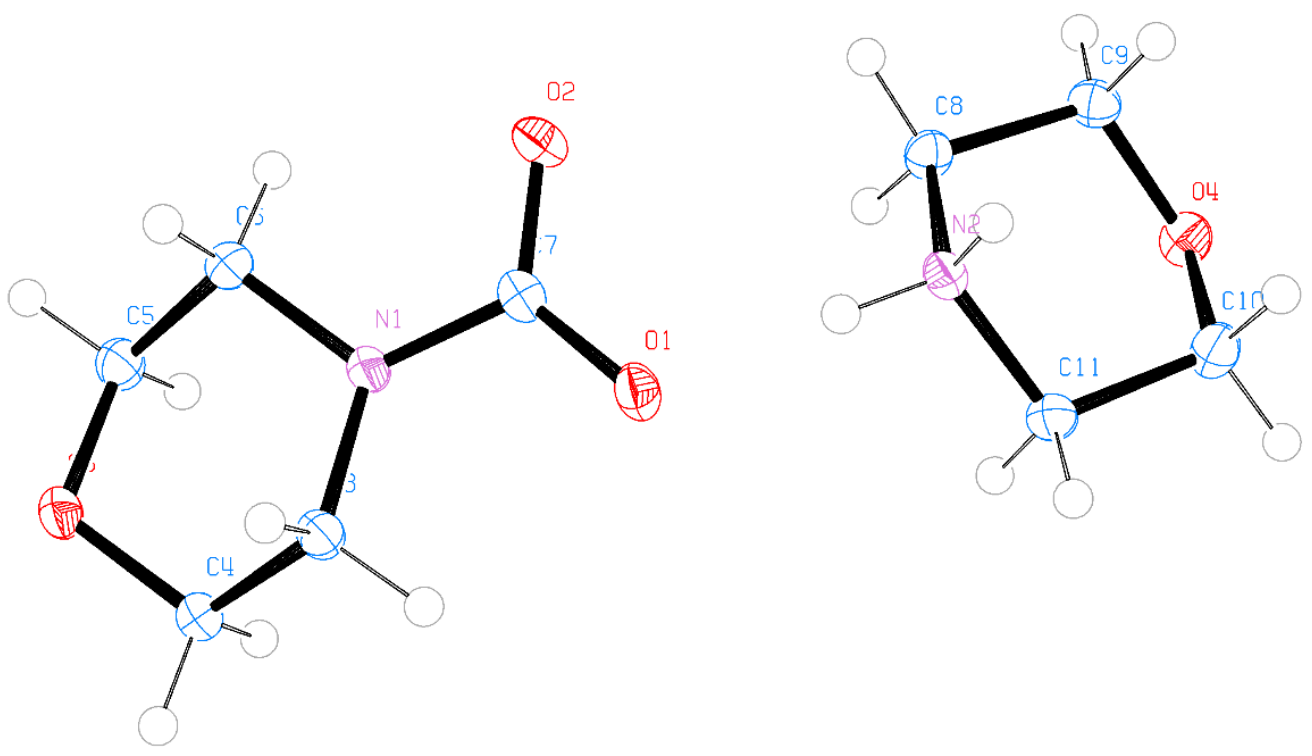

Figure S1: Molecular structure of morphylammonium morphylcarbamate with ellipsoids at $50 \%$ probability. Select bond distances, $\AA$ : $(\mathrm{C} 7)-(\mathrm{O} 1)=1.27,(\mathrm{C} 7)-(\mathrm{O} 2)=1.26,(\mathrm{C} 7)-(\mathrm{N} 1)=$ 1.39 . 


\section{Optimization Tables for $\mathrm{CO}_{2}$ Hydrogenation:}

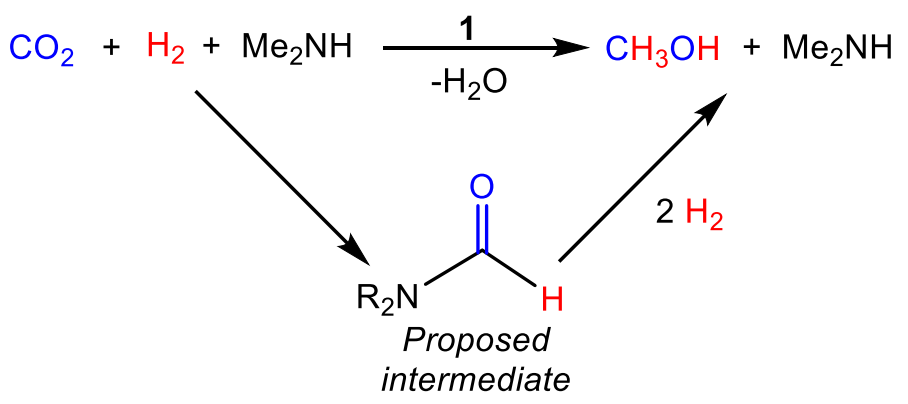

Table S1: Reactions Related to using Dimethylamine as a Shuttle

\begin{tabular}{|c|cc|cccc|}
\hline Entry $^{\mathbf{b}}$ & $\begin{array}{c}\text { Substrate } \\
(\mathbf{m m o l})\end{array}$ & $\begin{array}{c}\mathbf{C O}_{2} / \mathbf{H}_{2} \\
(\mathbf{p s i})\end{array}$ & $\begin{array}{c}\mathbf{D M F}^{\mathbf{c}} \\
(\mathbf{m m o l})\end{array}$ & $\begin{array}{c}\mathbf{C H}_{3} \mathbf{O H} \\
(\mathbf{m m o l})\end{array}$ & $\begin{array}{c}\mathbf{C o n v}^{\mathbf{d}} \\
(\%)\end{array}$ & TON $^{\mathrm{\%}}$ \\
\hline $1^{\mathrm{a}}$ & $\begin{array}{c}\mathrm{HNMe}_{2} \\
(21)\end{array}$ & $100 / 900$ & 5.22 & - & $25 \%$ & 0 \\
\hline $2^{\mathrm{a}, \mathrm{f}}$ & $\begin{array}{c}\mathrm{HNMe}_{2} \\
(21)\end{array}$ & $100 / 900$ & 6.65 & - & $32 \%$ & 0 \\
\hline $3^{\mathrm{g}}$ & $\begin{array}{c}\mathrm{DMF} \\
(0.2)\end{array}$ & $0 / 900$ & 0.01 & 0.1 & $95 \%^{\mathrm{h}}$ & 40 \\
\hline $4^{\mathrm{g}}$ & $\begin{array}{c}\mathrm{DMF} \\
(0.2)\end{array}$ & $100 / 900$ & 0.2 & - & $0 \% \%^{\mathrm{h}}$ & 0 \\
\hline
\end{tabular}

aReaction conditions: $21 \mathrm{mmol}$ dimethylamine, 5 mol 1 (0.02 mol\%), 0.1 mmol LiOTf, 6 mL dioxane, 100 psi $\mathrm{CO}_{2}, 900$ psi $\mathrm{H}_{2}, 120{ }^{\circ} \mathrm{C}, 16 \mathrm{hrs}$. ${ }^{\mathrm{b}}$ Each entry represents only one trial unless otherwise indicated. ${ }^{\mathrm{c}} \mathrm{DMF}=$ dimethylformamide. ${ }^{\mathrm{d} C o n v e r s i o n}$ is tabulated with respect to DMF production unless otherwise indicated. ${ }^{\mathrm{e}} \mathrm{TON}$ quantified by GC-MS, tabulated as moles of product/moles of 1 in each step. ${ }^{\mathrm{f}} 15 \mu \mathrm{mol} 1\left(0.07\right.$ mol\%). ${ }^{\mathrm{g}} \mathrm{Reaction}$ conditions: $0.2 \mathrm{mmol}$ DMF, $2.5 \mu \mathrm{mol} 1$ (1.3 mol\%), $0.1 \mathrm{mmol} \mathrm{LiOTf,} 5 \mathrm{~mL}$ dioxane, X psi $\mathrm{CO}_{2}, 900$ psi $\mathrm{H}_{2}, 120$ ${ }^{\circ} \mathrm{C}, 16 \mathrm{hrs}$. ${ }^{\mathrm{h}}$ Conversion is tabulated with respect to DMF consumption. 
Table S2: Two-Step Reactions- Effect of Morphylammonium Morphylcarbamate

\begin{tabular}{|c|cc|cccc|c|}
\hline Entry $^{\mathrm{a}}$ & $\begin{array}{c}\text { Substrate } \\
(\mathbf{m m o l})\end{array}$ & $\begin{array}{c}\mathbf{C O}_{2} / \mathbf{H}_{2} \\
(\mathbf{p s i})\end{array}$ & $\begin{array}{c}\mathbf{F M}^{\mathbf{c}} \\
(\mathbf{m m o l})\end{array}$ & $\begin{array}{c}\mathbf{C H}_{3} \mathbf{O H} \\
(\mathbf{m m o l})\end{array}$ & $\begin{array}{c}\mathbf{C o n v}^{\mathbf{d}} \\
(\mathbf{\%})\end{array}$ & TON $^{\mathrm{e}}$ & Additive \\
\hline $2 \mathrm{~A}^{\mathrm{b}}$ & $\begin{array}{c}\text { Morpholine } \\
(21)\end{array}$ & $100 / 900$ & 4.87 & - & $24 \%$ & 330 & - \\
$2 \mathrm{~B}^{\mathrm{b}}$ & $\mathrm{FM}(1.25)$ & $0 / 900$ & 0.44 & 0.81 & $65 \%$ & 80 & - \\
\hline $5 \mathrm{~A}$ & $\begin{array}{c}\text { Morpholine } \\
(21)\end{array}$ & $100 / 900$ & 5.94 & - & $29 \%$ & 400 & - \\
$5 \mathrm{~B}$ & $\mathrm{FM}(1.25)$ & $0 / 900$ & 1.21 & 0.04 & $3 \%$ & 4 & $\begin{array}{c}\text { Carbamate } \\
(1.25 \\
\text { mmol) }\end{array}$ \\
\hline $6 \mathrm{~A}^{\mathrm{f}}$ & $\begin{array}{c}\text { Carbamate } \\
(3.00) \\
\text { Carbamate } \\
(1.25)\end{array}$ & $100 / 900$ & 0.64 & - & $3 \%$ & 40 & - \\
$6 \mathrm{~B}^{\mathrm{f}}$ & $0 / 900$ & 0.11 & - & $8 \%$ & 10 & - \\
\hline
\end{tabular}

Reaction conditions: Step A- $21 \mathrm{mmol}$ morpholine, $2.02 \mathrm{~g} 3 \AA$ molecular sieves, $15 \mu \mathrm{mol} 1$ ( $0.07 \mathrm{~mol} \%), 8 \mathrm{~mL}$ dioxane, 100 psi $\mathrm{CO}_{2}, 900$ psi $\mathrm{H}_{2}, 120{ }^{\circ} \mathrm{C}, 16 \mathrm{hrs}$; Step B- 1.25 mmol FM from step A solution, $10 \mu \mathrm{mol} 1(0.8$ mol\%), $1 \mathrm{mmol}$ LiOTf, $2.5 \mathrm{mmol} \mathrm{DBU}$, with or without $1.25 \mathrm{mmol}$ morphylammonium morphylcarbamate in 5 $\mathrm{mL}$ total volume of solution, $100 \mathrm{psi} \mathrm{H}_{2}, 120{ }^{\circ} \mathrm{C}, 16 \mathrm{hrs}$. ${ }^{\mathrm{a}} \mathrm{Each}$ entry represents only one trial unless otherwise indicated. ${ }^{b}$ Average of two trials. ${ }^{c} \mathrm{FM}=$ formylmorpholine. ${ }^{\mathrm{d}}$ For step A conversion is tabulated with respect to FM production as no other products were detected. For step B conversion is tabulated with respect to FM consumption. ${ }^{\mathrm{e}} \mathrm{TON}$ quantified by GC-MS, tabulated as moles of product/moles of $\mathbf{1}$ in each step. ${ }^{\mathrm{f}} \mathrm{Carbamate}$ used as the substrate in both steps; step B conversion and TON calculated with respect to FM production. 
Table S3: Two-Step Reactions- Effect of LiOTf

\begin{tabular}{|c|c|c|c|c|c|c|c|}
\hline Entry $^{a}$ & $\begin{array}{c}\text { Substrate } \\
(\mathrm{mmol})\end{array}$ & $\begin{array}{c}\mathrm{CO}_{2} / \mathrm{H}_{2} \\
(\mathrm{psi})\end{array}$ & $\begin{array}{c}\text { FMc }^{\mathbf{c}} \\
(\mathrm{mmol})\end{array}$ & $\begin{array}{c}\mathrm{CH}_{3} \mathrm{OH} \\
(\mathrm{mmol})\end{array}$ & $\begin{array}{c}\text { Conv. }^{d} \\
(\%)\end{array}$ & TON $^{\mathrm{e}}$ & Additive \\
\hline $2 \mathrm{~A}^{\mathrm{b}}$ & $\begin{array}{l}\text { Morpholine } \\
\text { (21) }\end{array}$ & $100 / 900$ & 4.87 & - & $24 \%$ & 330 & - \\
\hline $2 \mathrm{~B}^{\mathrm{b}}$ & $\mathrm{FM}(1.25)$ & $0 / 900$ & 0.44 & 0.81 & $65 \%$ & 80 & $\begin{array}{c}\text { LiOTf } \\
(1 \mathrm{mmol})\end{array}$ \\
\hline $7 \mathrm{~A}$ & $\begin{array}{l}\text { Morpholine } \\
\text { (21) }\end{array}$ & $100 / 900$ & 5.23 & - & $25 \%$ & 350 & - \\
\hline $7 \mathrm{~B}$ & $\mathrm{FM}(1.25)$ & 0/900 & 1.17 & 0.08 & $6 \%$ & 10 & - \\
\hline $8 \mathrm{~A}$ & $\begin{array}{l}\text { Morpholine } \\
\text { (21) }\end{array}$ & $100 / 900$ & 1.86 & - & $9 \%$ & 120 & $\begin{array}{c}\text { LiOTf } \\
(1 \mathrm{mmol})\end{array}$ \\
\hline $8 B$ & $\mathrm{FM}(1.25)$ & $0 / 900$ & 0.28 & 1.07 & $78 \%$ & 110 & $\begin{array}{l}\text { LiOTf } \\
(1 \mathrm{mmol})\end{array}$ \\
\hline
\end{tabular}

Reaction conditions: Step A- 21 mmol morpholine with or without 1 mmol LiOTf, $2.02 \mathrm{~g} 3 \AA$ molecular sieves, $15 \mu \mathrm{mol} 1(0.07 \mathrm{~mol} \%), 8 \mathrm{~mL}$ dioxane, $100 \mathrm{psi} \mathrm{CO}_{2}, 900 \mathrm{psi} \mathrm{H}_{2}, 120^{\circ} \mathrm{C}, 16 \mathrm{hrs}$; Step B- $1.25 \mathrm{mmol}$ FM from step A solution, $10 \mu \mathrm{mol} 1(0.8 \mathrm{~mol} \%)$ with or without $1 \mathrm{mmol} \mathrm{LiOTf}, 2.5 \mathrm{mmol} \mathrm{DBU}$, in $5 \mathrm{~mL}$ total volume of solution, $100 \mathrm{psi}_{2}, 120^{\circ} \mathrm{C}, 16 \mathrm{hrs}$. ${ }^{\mathrm{a}} \mathrm{Each}$ entry represents only one trial unless otherwise indicated. ${ }^{\mathrm{b}}$ Average of two trials. ${ }^{\mathrm{F}} \mathrm{FM}=$ formylmorpholine. ${ }^{\mathrm{d}}$ For step A conversion is tabulated with respect to FM production as no other

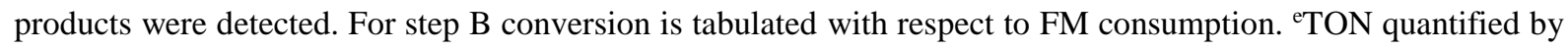
GC-MS, tabulated as moles of product/moles of $\mathbf{1}$ in each step. 
Table S4: Two-Step Reactions- Effect of DBU

\begin{tabular}{|c|c|c|c|c|c|c|c|}
\hline Entry $^{a}$ & $\begin{array}{c}\begin{array}{c}\text { Substrate } \\
(\mathrm{mmol})\end{array} \\
\end{array}$ & $\begin{array}{c}\mathrm{CO}_{2} / \mathbf{H}_{2} \\
(\mathrm{psi})\end{array}$ & $\begin{array}{c}\mathbf{F M}^{\mathbf{c}} \\
(\mathbf{m m o l})\end{array}$ & $\begin{array}{l}\mathrm{CH}_{3} \mathrm{OH} \\
\text { (mmol) }\end{array}$ & $\begin{array}{c}\text { Conv. }^{\mathrm{d}} \\
(\%)\end{array}$ & TON & Additive \\
\hline $1 \mathrm{~A}^{\mathrm{b}}$ & $\begin{array}{l}\text { Morpholine } \\
\text { (21) }\end{array}$ & $100 / 900$ & 4.87 & - & $24 \%$ & 330 & - \\
\hline $1 \mathrm{~B}^{\mathrm{b}}$ & FM (1.25) & $0 / 900$ & 0.44 & 0.81 & $65 \%$ & 80 & $\begin{array}{c}\mathrm{DBU} \\
(2.5 \mathrm{mmol})\end{array}$ \\
\hline $9 \mathrm{~A}$ & $\begin{array}{l}\text { Morpholine } \\
\text { (21) }\end{array}$ & $100 / 900$ & 5.03 & - & $24 \%$ & 340 & - \\
\hline 9B & FM (1.25) & $0 / 900$ & 1.38 & - & $0 \%$ & 0 & - \\
\hline $10 A^{b}$ & $\begin{array}{l}\text { Morpholine } \\
\text { (21) }\end{array}$ & $100 / 900$ & 11.90 & - & $56 \%$ & 790 & $\begin{array}{c}\text { DBU } \\
(2.5 \mathrm{mmol})\end{array}$ \\
\hline $10 \mathrm{~B}^{\mathrm{b}}$ & $\mathrm{FM}(1.25)$ & $0 / 900$ & 0.32 & 0.55 & $74 \%$ & 60 & $\begin{array}{c}\mathrm{DBU} \\
(2.5 \mathrm{mmol})\end{array}$ \\
\hline $11 \mathrm{~A}^{\mathrm{b}}$ & $\begin{array}{l}\text { Morpholine } \\
\text { (21) }\end{array}$ & $100 / 900$ & 4.80 & - & $23 \%$ & 320 & - \\
\hline $11 \mathrm{~B}^{\mathrm{b}, \mathrm{f}}$ & $\mathrm{FM}(1.25)$ & $0 / 900$ & 0.01 & 0.84 & $>99 \%$ & 80 & $\begin{array}{c}\text { DBU } \\
(5 \mathrm{mmol})\end{array}$ \\
\hline $12 \mathrm{~A}^{\mathrm{b}, \mathrm{f}}$ & $\begin{array}{l}\text { Morpholine } \\
\text { (21) }\end{array}$ & $100 / 900$ & 9.16 & - & $44 \%$ & 610 & $\begin{array}{c}\text { DBU } \\
(5 \mathrm{mmol})\end{array}$ \\
\hline $12 \mathrm{~B}^{\mathrm{b}, \mathrm{f}}$ & FM (1.25) & $0 / 900$ & 1.77 & - & $0 \%$ & 0 & - \\
\hline
\end{tabular}

Reaction conditions: Step A- 21 mmol morpholine with or without 2.5 or 5 mmol DBU, $2.02 \mathrm{~g} 3 \AA$ molecular sieves, $15 \mu \mathrm{mol} 1(0.07 \mathrm{~mol} \%), 8 \mathrm{~mL}$ dioxane, $100 \mathrm{psi} \mathrm{CO}$, $900 \mathrm{psi} \mathrm{H}_{2}, 120^{\circ} \mathrm{C}, 16 \mathrm{hrs}$; Step B- $1.25 \mathrm{mmol}$ FM from step A solution, $10 \mu \mathrm{mol} 1(0.8 \mathrm{~mol} \%), 1 \mathrm{mmol}$ LiOTf with or without 2.5 or $5 \mathrm{mmol} \mathrm{DBU}$, in $5 \mathrm{~mL}$ total volume of solution, $100 \mathrm{psi} \mathrm{H}_{2}, 120^{\circ} \mathrm{C}, 16 \mathrm{hrs}$. ${ }^{a}$ Each entry represents only one trial unless otherwise indicated. ${ }^{\mathrm{b}}$ Average of two trials. ${ }^{\mathrm{c}} \mathrm{FM}=$ formylmorpholine. ${ }^{\mathrm{d}} \mathrm{For}$ step A conversion is tabulated with respect to FM production as no other

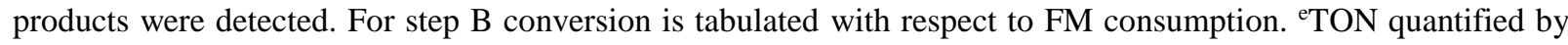

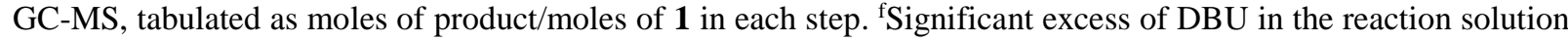
interfered with quantification of FM by GC-MS. 
Table S5: Two-Step Reactions- Effect of Solvent

\begin{tabular}{|c|c|c|c|c|c|c|c|}
\hline Entry $^{a}$ & $\begin{array}{c}\text { Substrate } \\
(\mathbf{m m o l})\end{array}$ & $\begin{array}{c}\mathrm{CO}_{2} / \mathbf{H}_{2} \\
(\mathrm{psi})\end{array}$ & $\begin{array}{c}\mathbf{F M}^{\mathbf{b}} \\
(\mathbf{m m o l})\end{array}$ & $\begin{array}{l}\mathrm{CH}_{3} \mathrm{OH} \\
(\mathrm{mmol})\end{array}$ & $\begin{array}{c}\text { Conv. }^{c} \\
(\%)\end{array}$ & TON ${ }^{d}$ & Solvent \\
\hline $1 \mathrm{~A}$ & $\begin{array}{l}\text { Morpholine } \\
\text { (21) }\end{array}$ & $100 / 900$ & 4.87 & - & $24 \%$ & 330 & $\begin{array}{c}1,4- \\
\text { dioxane }\end{array}$ \\
\hline 1B & FM (1.25) & $0 / 900$ & 0.44 & 0.81 & $65 \%$ & 80 & \\
\hline $13 \mathrm{~A}$ & $\begin{array}{l}\text { Morpholine } \\
\text { (21) }\end{array}$ & $100 / 900$ & 4.42 & - & $21 \%$ & 290 & $\mathrm{THF}^{\mathrm{e}}$ \\
\hline $13 \mathrm{~B}$ & FM (1.25) & $0 / 900$ & 0.01 & 1.13 & $>99 \%$ & 110 & \\
\hline $14 \mathrm{~A}$ & $\begin{array}{l}\text { Morpholine } \\
\text { (21) }\end{array}$ & $100 / 900$ & 3.30 & - & $16 \%$ & 220 & Toluene \\
\hline 14B & FM (1.25) & $0 / 900$ & 0.01 & 1.29 & $>99 \%$ & 130 & \\
\hline $15 \mathrm{~A}$ & $\begin{array}{l}\text { Morpholine } \\
\text { (21) }\end{array}$ & $100 / 900$ & 3.37 & - & $16 \%$ & 220 & $\begin{array}{l}\text { Ethyl } \\
\text { Acetate }\end{array}$ \\
\hline $15 B$ & FM (1.25) & $0 / 900$ & 0.09 & 0.37 & $93 \%$ & 40 & \\
\hline
\end{tabular}

Reaction conditions: Step A- $21 \mathrm{mmol}$ morpholine, $2.02 \mathrm{~g} 3 \AA$ molecular sieves, $15 \mu \mathrm{mol} 1(0.07 \mathrm{~mol} \%), 8 \mathrm{~mL}$ solvent, 100 psi $\mathrm{CO}_{2}, 900$ psi $\mathrm{H}_{2}, 120^{\circ} \mathrm{C}, 16 \mathrm{hrs}$; Step B- 1.25 mmol FM from step A solution, $10 \mu \mathrm{mol} 1(0.8$ mol\%), 1 mmol LiOTf, $2.5 \mathrm{mmol}$ DBU in $5 \mathrm{~mL}$ total volume of solution, $100 \mathrm{psi} \mathrm{H}_{2}, 120{ }^{\circ} \mathrm{C}, 16 \mathrm{hrs} .{ }^{\mathrm{a}}$ Each entry represents an average of two trials unless otherwise indicated. ${ }^{b} \mathrm{FM}=$ formylmorpholine. ${ }^{\mathrm{c}} \mathrm{For}$ step A conversion is tabulated with respect to FM production as no other products were detected. For step B conversion is tabulated with respect to FM consumption. ${ }^{\mathrm{d}} \mathrm{TON}$ quantified by GC-MS/GC-FID, tabulated as moles of product/moles of 1 in each step. ${ }^{\mathrm{e}} \mathrm{THF}=$ tetrahydrofuran. 
Table S6: Two-Step Reactions- Effect of Pressure

\begin{tabular}{|c|cc|cccc|}
\hline Entry $^{\mathbf{a}}$ & $\begin{array}{c}\text { Substrate } \\
(\mathbf{m m o l})\end{array}$ & $\begin{array}{c}\mathbf{C O}_{2} / \mathbf{H}_{2} \\
(\mathbf{p s i})\end{array}$ & $\begin{array}{c}\mathbf{F M}^{\mathbf{b}} \\
(\mathbf{m m o l})\end{array}$ & $\begin{array}{c}\mathbf{C H}_{3} \mathbf{O H} \\
(\mathbf{m m o l})\end{array}$ & $\begin{array}{c}\mathbf{C o n v}^{\mathbf{c}} \\
(\mathbf{\%})\end{array}$ & TON $^{\mathbf{d}}$ \\
\hline $13 \mathrm{~A}$ & $\begin{array}{c}\text { Morpholine } \\
(21)\end{array}$ & $100 / 900$ & 4.42 & - & $21 \%$ & 290 \\
$13 \mathrm{~B}$ & $\mathrm{FM}(1.25)$ & $0 / 900$ & 0.01 & 1.13 & $>99 \%$ & 110 \\
\hline $16 \mathrm{~A}$ & $\begin{array}{c}\text { Morpholine } \\
(21)\end{array}$ & $200 / 800$ & 6.91 & - & $33 \%$ & 460 \\
$16 \mathrm{~B}$ & $\mathrm{FM}(1.25)$ & $0 / 800$ & 0.01 & 1.08 & $>99 \%$ & 110 \\
\hline $17 \mathrm{~A}$ & $\begin{array}{c}\text { Morpholine } \\
(21)\end{array}$ & $200 / 1200$ & 9.24 & - & $44 \%$ & 620 \\
$17 \mathrm{~B}$ & $\mathrm{FM}(4.00)$ & $0 / 1200$ & 0.05 & 3.40 & $99 \%$ & 340 \\
\hline $18 \mathrm{~A}^{\mathrm{e}}$ & $\begin{array}{c}\text { Morpholine } \\
(21)\end{array}$ & $200 / 1200$ & 10.22 & - & $49 \%$ & 680 \\
$18 \mathrm{~B}^{\mathrm{e}}$ & $\mathrm{FM}(4.00)$ & $0 / 1200$ & 3.82 & - & $5 \%$ & 0 \\
\hline
\end{tabular}

Reaction conditions: Step A- 21 mmol morpholine, 2.02 g $3 \AA$ molecular sieves, $15 \mu \mathrm{mol} 1$ (0.07 mol\%), 8 $\mathrm{mL}$ THF, X psi $\mathrm{CO}_{2}$, Y psi $\mathrm{H}_{2}, 120^{\circ} \mathrm{C}, 16 \mathrm{hrs}$; Step B- 1.25 or $4.00 \mathrm{mmol}$ FM from step A solution, $10 \mu \mathrm{mol}$

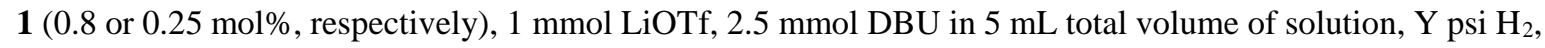
$120{ }^{\circ} \mathrm{C}, 16 \mathrm{hrs}$. ${ }^{\mathrm{a}}$ Each entry represents an average of two trials unless otherwise indicated. ${ }^{\mathrm{b}} \mathrm{FM}=$ formylmorpholine. ${ }^{c}$ For step A conversion is tabulated with respect to FM production as no other products

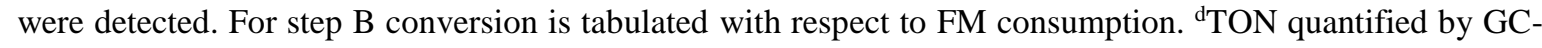

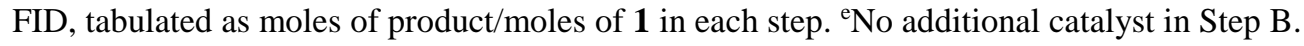


Table S7: Two-Step Reactions- Varying Additive Amounts

\begin{tabular}{|c|c|c|c|c|c|c|c|}
\hline Entry $^{a}$ & $\begin{array}{l}\text { Substrate } \\
(\mathrm{mmol})\end{array}$ & $\begin{array}{c}\mathrm{CO}_{2} / \mathrm{H}_{2} \\
(\mathbf{p s i})\end{array}$ & $\begin{array}{c}\text { FM }^{\mathbf{b}} \\
(\mathbf{m m o l})\end{array}$ & $\begin{array}{c}\mathrm{CH}_{3} \mathrm{OH} \\
(\mathrm{mmol})\end{array}$ & $\begin{array}{c}\text { Conv. } \\
(\%)\end{array}$ & TON $^{d}$ & $\begin{array}{c}\text { LiOTf, } \\
\text { DBU } \\
\text { (mmol) }\end{array}$ \\
\hline $17 \mathrm{~A}$ & $\begin{array}{l}\text { Morpholine } \\
\text { (21) }\end{array}$ & $200 / 1200$ & 9.24 & - & $44 \%$ & 620 & - \\
\hline 17B & FM (4.00) & $0 / 1200$ & 0.05 & 3.40 & $99 \%$ & 340 & $1.0,2.5$ \\
\hline $19 \mathrm{~A}$ & $\begin{array}{l}\text { Morpholine } \\
\text { (21) }\end{array}$ & $200 / 1200$ & 9.19 & - & $44 \%$ & 610 & - \\
\hline $19 \mathrm{~B}$ & $\mathrm{FM}(4.00)$ & $0 / 1200$ & 0.20 & 3.27 & $95 \%$ & 330 & $1.0,4.0$ \\
\hline $20 \mathrm{~A}$ & $\begin{array}{l}\text { Morpholine } \\
\text { (21) }\end{array}$ & $200 / 1200$ & 9.29 & - & $44 \%$ & 620 & - \\
\hline $20 \mathrm{~B}$ & FM (4.00) & $0 / 1200$ & 0.09 & 3.41 & $98 \%$ & 340 & $1.5,2.5$ \\
\hline $21 \mathrm{~A}$ & $\begin{array}{l}\text { Morpholine } \\
\text { (21) }\end{array}$ & $200 / 900$ & 9.28 & - & $44 \%$ & 620 & - \\
\hline $21 B$ & FM (4.00) & $0 / 1200$ & 0.12 & 3.20 & $97 \%$ & 320 & $1.5,4.0$ \\
\hline
\end{tabular}

Reaction conditions: Step A- $21 \mathrm{mmol}$ morpholine, $2.02 \mathrm{~g} 3 \AA$ A molecular sieves, $15 \mu \mathrm{mol} 1(0.07 \mathrm{~mol} \%), 8 \mathrm{~mL}$ THF, 200 psi CO 2,1200 psi $\mathrm{H}_{2}, 120^{\circ} \mathrm{C}, 16 \mathrm{hrs}$; Step B- 4.00 mmol FM from step A solution, $10 \mu \mathrm{mol} 1(0.25$ mol\%), X mmol LiOTf, Y mmol DBU in $5 \mathrm{~mL}$ total volume of solution, $1200 \mathrm{psi} \mathrm{H}_{2}, 120{ }^{\circ} \mathrm{C}, 16 \mathrm{hrs}$. ${ }^{\mathrm{a}}$ Each entry represents an average of two trials unless otherwise indicated. ${ }^{b} \mathrm{FM}=$ formylmorpholine. ${ }^{\mathrm{c}} \mathrm{For}$ step A conversion is tabulated with respect to FM production as no other products were detected. For step B conversion is tabulated with respect to FM consumption. ${ }^{\mathrm{d}}$ TON quantified by GC-FID, tabulated as moles of product/moles of $\mathbf{1}$ in each step. 
Table S8: Two-Step Reactions- Effect of Temperature

\begin{tabular}{|c|cc|cccc|c|}
\hline Entry $^{\mathbf{a}}$ & $\begin{array}{c}\text { Substrate } \\
(\mathbf{m m o l})\end{array}$ & $\begin{array}{c}\mathbf{C O}_{2} / \mathbf{H}_{2} \\
(\mathbf{p s i})\end{array}$ & $\begin{array}{c}\mathbf{F M}^{\mathbf{b}} \\
(\mathbf{m m o l})\end{array}$ & $\begin{array}{c}\mathbf{C H}_{3} \mathbf{O H} \\
(\mathbf{m m o l})\end{array}$ & $\begin{array}{c}\mathbf{C o n v}^{\mathbf{c}} \\
(\mathbf{\%})\end{array}$ & TON $^{\mathbf{d}}$ & Temp. $^{\left.{ }^{\mathbf{o}} \mathbf{C}\right)}$ \\
\hline 17A & $\begin{array}{c}\text { Morpholine } \\
(21)\end{array}$ & $200 / 1200$ & 9.24 & - & $44 \%$ & 620 & 120 \\
17B & FM (4.00) & $0 / 1200$ & 0.05 & 3.40 & $99 \%$ & 340 & 120 \\
\hline 22A & $\begin{array}{c}\text { Morpholine } \\
(21)\end{array}$ & $200 / 1200$ & 16.76 & - & $80 \%$ & 1120 & 100 \\
22B & FM (4.00) & $0 / 1200$ & 0.01 & 3.60 & $>99 \%$ & 360 & 100 \\
\hline 23A & $\begin{array}{c}\text { Morpholine } \\
(21)\end{array}$ & $200 / 1200$ & 15.24 & - & $73 \%$ & 1020 & 80 \\
23B & FM (4.00) & $0 / 1200$ & 0.44 & 3.20 & $89 \%$ & 320 & 80 \\
\hline
\end{tabular}

Reaction conditions: Step A- 21 mmol morpholine, $2.02 \mathrm{~g} 3 \AA$ molecular sieves, $15 \mu \mathrm{mol} 1$ (0.07 mol\%), $8 \mathrm{~mL}$ THF, 200 psi $\mathrm{CO}_{2}, 1200$ psi $\mathrm{H}_{2}, \mathrm{X}^{\circ} \mathrm{C}, 16 \mathrm{hrs}$; Step B- $4.00 \mathrm{mmol}$ FM from step A solution, $10 \mu \mathrm{mol} 1$ (0.25 mol\%), 1 mmol LiOTf, 2.5 mmol DBU in $5 \mathrm{~mL}$ total volume of solution, $1200 \mathrm{psi}_{2}, \mathrm{X}^{\circ} \mathrm{C}, 16$ hrs. ${ }^{\mathrm{a}}$ Each entry represents an average of two trials unless otherwise indicated. ${ }^{b} \mathrm{FM}=$ formylmorpholine. ${ }^{\mathrm{c}}$ For step A conversion is tabulated with respect to FM production as no other products were detected. For step B conversion is tabulated with respect to FM consumption. ${ }^{\mathrm{d}}$ TON quantified by GC-FID, tabulated as moles of product/moles of $\mathbf{1}$ in each step. 